\title{
Linx
}

Revue des linguistes de l'université Paris X Nanterre

$57 \mid 2007$

Études de syntaxe : français parlé, français hors de

France, créoles

\section{Discours rapporté et particules}

Julie Peuvergne

\section{(2) OpenEdition}

Journals

Édition électronique

URL : http://journals.openedition.org/linx/67

DOI : $10.4000 /$ linx. 67

ISSN : 2118-9692

Éditeur

Presses universitaires de Paris Nanterre

Édition imprimée

Date de publication : 1 décembre 2007

Pagination : 123-131

ISSN : 0246-8743

\section{Référence électronique}

Julie Peuvergne, «Discours rapporté et particules », Linx [En ligne], 57 | 2007, mis en ligne le 15 février 2011, consulté le 15 mai 2020. URL : http://journals.openedition.org/linx/67 ; DOI : https://doi.org/ 10.4000/linx.67 


\title{
Discours rapporté et particules
}

\author{
Julie Peuvergne \\ Université Paris Ouest Nanterre La Défense et MoDyCo \\ Albert-Ludwig Universität, Freiburg (Allemagne)
}

Les données présentées ici ont été recueillies en 2007 et 2008, à Yaoundé et à Douala, dans le cadre du projet CIEL-F1. Cette étude du discours rapporté (désormais DR) s'inscrit dans un questionnement plus large sur le morphème que. Pour ce qui concerne le DR dans le français au Cameroun, nous renvoyons à des indications chiffrées données par Queffélec (2006) : «Introduisant le DR dans plus de $85 \%$ des occurrences, dire est de loin le verbe recteur le plus couramment employé. [...] $64 \%$ des occurrences de DR sont introduites par un que, pourcentage très supérieur à celui qu'Italia avait relevé chez des non-scolarisés gabonais (25\%)» (pp. 270-271). Ce n'est

\footnotetext{
${ }^{1}$ CIEL_F $=$ Corpus International Ecologique de la Langue Française, initiative franco-belgoallemande associant les Universités de Lyon II, Paris Ouest Nanterre la Défense, Louvain-la-Neuve, Freiburg-im-Breisgau et Halle-Wittenberg. Ce projet a pour objectif de constituer un vaste corpus sur les français dans le monde, selon des critères relevant d'une part d'une approche des activités interactionnelles dans des circonstances écologiques, d'autre part d'une typologie des aires et situations d'usages.

La typologie des situations prévoit le recueil de conversations ordinaires, d'émissions de radio et d'interactions en situation de travail. http://ciel-f.org/. Nous avons ici exploité pour l'essentiel des interactions ordinaires entre amis ou en famille, lors desquelles nous sommes généralement présente, mais sans avoir pour autant suscité les conversations.
} 
ainsi pas la présence de que qui s'avère caractéristique des données de notre corpus, mais plutôt son extrême fréquence dans le DR.

D'autres marqueurs signalent aussi l'insertion d'une nouvelle énonciation: pauses, prosodie, particules. C'est sur la base de cette dernière catégorie que nous allons envisager le faisceau d'indices signalant le DR. Nous verrons que les particules jouent des rôles multiples, structurant le DR sur le modèle de la conversation spontanée, mais signalant également l'intervention du locuteur dans les propos qu'il rapporte. Les rapports qu'elles entretiennent avec le verbe de parole et leur participation à la prédication nous permettront de proposer des éléments d'interprétation quant au rôle de que.

\section{Hétérogénéité terminologique}

De façon générale, le terme de «discours rapporté » désigne «le recours à une apparence d'énonciation venue d'ailleurs » (Vincent \& Dubois, 1997, p. 12).

Le DR se caractérise par le fait qu'un locuteur $(\mathrm{L})$, engagé dans la construction d'un discours (qu'on appellera «discours global») convoque la parole d'autrui (de locuteur(s), désignés en $l_{1}, l_{2} \ldots$ ) relevant d'une autre situation d'énonciation (situation représentée). Nous utiliserons le terme de "segment introducteur», comportant les « indications explicitées par lesquelles $\mathrm{L}$ reconstitue, pour ce qu'il cite, une situation d'énonciation » (Authier, 1978, p. 52). Ce segment comporte au minimum un verbe de parole, et se différencie du discours cité (désormais DCé), qui est donné comme ayant été produit par 1 .

Les particules constituent une liste ouverte de termes (bon, mais, oui, non..., interjections...) qui viennent s'insérer entre le segment introducteur et le DCé. La question de leur appartenance à l'un ou l'autre discours, lorsqu'elle est discutée, dépend principalement des fonctions attribuées aux particules. Celles-ci constituent seulement des «balises», indices d'entrée dans le DR ne faisant pas partie du DCé, pour Italia (2005, p. 198), ce sont des « ligateurs » chez Morel et Danon-Boileau (1998, p. 130). Pour Vincent (1989), les particules d'attaque de réplique contribuent à signaler les changements de tours de parole, à lier les éléments du dialogue entre eux, et elles relèvent en ce sens du DR (p. 597). Elles s'opposent ainsi aux connecteurs (ça fait que, alors, puis...), qui lient l'action de dire au discours (ibid.). Fauré \& Vérine (2004) soulignent quant à eux la difficulté, dans certains cas, de rattacher les interjections au discours enchâssé ou enchâssant: elles sont à l'intersection du narratif et du dialogique, (p. 324).

Cette hétérogénéité des terminologies et des descriptions nous semble bien refléter la porosité de la frontière entre le discours de $\mathrm{L}$ et celui de 1 : «qu'il le veuille ou non, [L] tire toutes les ficelles de la situation d'énonciation qu'il rapporte et dans laquelle l'énoncé s'inscrit et prend son sens » (Authier, 1978, p. 53).

\section{Double structuration}

L'échange que nous allons étudier dans cette partie constitue un épisode de la narration d'une anecdote à visée argumentative, basée principalement sur une 
conversation rapportée. Le segment de départ de l'anecdote est « la fille là c'est une bonne folle », et c'est ce que JA va illustrer en reproduisant la conversation qu'elle a eue avec la personne en question. "Le dialogue est un jeu de mouvement [...]. Dans les conversations rapportées, on retrouve la représentation du même mouvement d'assertion / réplique mais qui se manifeste par l'alternance simulée - par différentes techniques - de locuteurs. » (Vincent, 1989, p. 595). Le DR est ici une représentation d'une situation passée, mais comme le soulignent Vincent \& Dubois: «Les paroles rapportées sont une approximation des paroles dites» (1997, p. 59). Elles doivent donc être analysées en tenant le plus grand compte de la situation dans laquelle elles ont été produites.

\subsection{Structuration en actes}

La conversation spontanée est structurée par les interactants grâce à divers procédés. Les «marqueurs de structuration de la conversation» (MSC) ont été identifiés, analysés et décrits entre autres par Auchlin (1981), qui les définit comme « une liste de morphèmes » (p. 88), qui structurent la conversation, et dont la signification « consiste en indications sur les niveaux de textualisation respectifs des segments - des constituants - affectés. » (p. 94). Les listes des MSC d'une part, des particules observables dans le DR d'autre part, présentent de nombreux points de recoupement. Nous allons maintenant tenter d'établir dans quelle mesure, au delà de leurs formes, leurs fonctions se recoupent également.

1-JA : a- après elle vient me suivre elle me dit que bon: tu es avec qui=

b- je dis que (je) suis avec mon homme/=

c- elle dit c'est ton gars/

d- je dis oui $\backslash$

e- (.) $a b:($.

f- au fait/ j'ai mon: (.) l'ami de mon: (.) mon gars là qui veut te voir lä\(-) $)^{2}$

Les éléments en italiques concernent elle (l $l_{1}$ ), par opposition à JA (qui est à la fois $L$ et $1_{2}$ ). Les retours à la ligne mettent en évidence les différents mouvements de l'échange, sur le modèle (ici simplifié) d'analyse de la conversation utilisé par Auchlin.

En a-, bon est certes marqueur de prise de parole (en plus de elle me dit que), mais il a aussi la fonction bien connue " de totaliser, d'intégrer une suite d'actes en un seul mouvement [...] qui sont rassemblés en un seul constituant», et ce, de façon rétroactive (en agissant sur les interventions du co-texte droit), ou proactive, comme c'est la cas ici (Auchlin, 1981, pp. 97-98). L'échange borné à gauche par bon, et à droite par l'interjection $a$ h, introduit le constituant f-. Celui-ci présente un changement de niveau textuel, signalé par an fait ${ }^{3}$. L'observation des MSC permet de considérer le passage a-/e- comme un échange préalable à fonction interactive (question/réponse, demande de confirmation/réponse-confirmation, réaction-évaluation), subordonné à

\footnotetext{
${ }^{2}$ Dans la transcription, nous mettons tout le passage en italiques, pour éviter de matérialiser une frontière entre segment introducteur et DCé (le DR étant considéré comme la somme des deux). 3 au fait couvre, au moins chez cette locutrice, les fonctions qu'on attribue généralement à en fait.
} 
f-, acte directeur d'information, qui se place à un niveau supérieur (voir l'exemple, relativement similaire, proposé par Auchlin, 1981, p. 91).

Dans l'échange tel qu'il est rapporté par JA, les MSC structurent le DR sur le modèle de la conversation spontanée. Cette structuration est partie intégrante de l'argumentation, qui consiste à présenter $l_{1}$ comme responsable de ces actes de conversation: donner une information ( $\mathrm{f}$ ) qui s'avère déplacée, par rapport aux éléments d'information de l'échange préalable qu'elle a initié ('bien que tu sois avec ton ami, je vais te présenter quelqu'un').

\subsection{Subjectivité du locuteur}

Certains indices ne peuvent être rapprochés d'une simulation de conversation spontanée, et opèrent donc au-delà de la tâche locale de mise en place du DR, organisant les différents éléments rapportés au niveau du discours global, selon leur importance pour l'argumentation.

On note, à partir de c-, un ralentissement progressif du débit et une diminution des indices de DR : a- comporte un segment introducteur étendu (connecteur, information narrative) ; à partir de b-, seul je dis que subsiste ; absence de que à partir de c-, et de dire en e-. La séquence dite préalable est en outre caractérisée par un débit rapide, une prosodie relativement plate, et un volume sonore bas. Ces indices intègrent cette partie de l'échange en un seul niveau de structuration, dont la clôture est matérialisée par l'interjection ah, encadrée de micro-pauses, et suivie du marqueur an fait.

Si une augmentation du nombre de marqueurs signale des discontinuités, des changements de niveaux de structuration ${ }^{4}$, l'absence de verbe de parole, amorcée par la diminution des indices de $\mathrm{DR}$, nous semble à ce titre significative : rompant le rythme mis en place, elle contribue à signaler une rupture. Cette discontinuité par soustraction, si l'on peut dire, a également pour effet d'assurer la cohésion entre les énoncés rapportés, en effaçant progressivement $\mathrm{L}$ au profit du discours cité, laissant en quelque sorte l'histoire parler d'elle-même. Mais comme le commente Authier (1978), «l'effacement ostentatoire de L [...] est un simulacre de non-intervention absolue de L dans son acte de rapport» (p. 51).

En e-, l'interjection ah marquant la réaction, elle désigne à elle seule le changement de tour de parole, liant ainsi plus fortement $\mathrm{f}$ - à ce qui précède. Fauré \& Vérine (2002) montrent que le choix des interjections dans le DR «semble relever autant, sinon plus, de la stratégie discursive » (p. 324). Pour le point de vue développé par L, l'argument (f-) n'a de valeur que dans son rapport avec l'échange préalable, et c'est précisément à cette jonction que les segments introducteurs (incursions explicites de $\mathrm{L}$, aussi minimes soient-elles) sont relayés par les particules qui, données comme produites par $l_{1}$, sont néanmoins les traces de la subjectivité de $\mathrm{L}$ dans l'organisation du discours.

Si l'analyse des particules en termes de MSC dans l'échange rapporté permet d'envisager une structuration en actes conversationnels, la prise en compte de l'ensemble des indices du DR montre qu'à ce fonctionnement local se superpose la structu-

\footnotetext{
${ }^{4}$ Voir à ce propos GÜLICH, 1999.
} 
ration du discours global. Le fait que les particules soient intégrées au discours cité ne doit pas masquer leur rôle dans l'élaboration du discours en cours, dont la visée est moins de re-produire une conversation passée que de présenter un point de vue. A côté de l'emploi massif de dire, introducteur neutre, les particules semblent au contraire être l'élément où le point de vue narratif se manifeste.

\subsection{Attitude modale}

La subjectivité de L se manifeste entre autres par l'attitude qu'il prête à l, et les particules peuvent alors s'avérer fondamentales pour l'argumentation. L'occurrence de DR dans l'exemple 2 prend place peu de temps après celle de l'exemple 1. Quant à l'exemple 3, le DR y est produit au cours d'une émission de radio sur la question de la soumission à l'autorité.

2-JA : a- on se retrouve:: [...]

$\mathrm{b}-$ je dis mais $\backslash(-)$

c- je suis/ en train de te dire que je suis avec mon gars/: toi tu me dis que/

(.) que je te retrouve comme ça comme ça/

d- après voilà le gars qui arrive (.)

3- IN : a- et il a insulté/ (-) le roi (dans sa chair) \(-)

b- quan:d on lui a dit que mais $\backslash(-)$

c- tu insultes le chef de ton peuple $=$

d- il s'est repenti/ (-) je ne savais pas que c'était le chef de mon peuple

La particule mais inscrit dans ces deux cas le DCé comme acte de réaction, plus précisément comme une évaluation négative de ce qui précède. La particule est ici investie pour orienter l'interprétation du rôle interlocutif des participants (Fauré \& Vérine, 2004, p. 325).

En 2, le DR marque la clôture d'un épisode de la narration (un nouveau s'ouvrant en d-), et la seule information nouvelle pour les interlocuteurs de JA est le fait qu'elle ait réagit verbalement. En effet, le DCé est une reprise thématique de l'échange représenté, et il semble donc que mais supporte seul la charge informationnelle : l'attitude modale de $l_{2}$, c'est-à-dire le jugement de valeur qui accompagne son discours ${ }^{5}$.

Les indices prosodiques ont pour effet d'intégrer mais au segment introducteur (courbe intégrante, pause), l'intonation finale descendante signalant son autonomie par rapport à ce qui suit (Meunier et al., 1991, p. 115). La solidarité que la particule entretient ainsi avec le verbe introducteur (renforcée par l'absence de que) invite à envisager la pause comme manifestant l'élaboration de deux programmes phrastiques distincts : le verbe introducteur et la particule d'une part, l'énoncé rapporté d'autre part.

\footnotetext{
${ }^{5} \mathrm{~L}$ et $\mathrm{l}_{2}$ étant ici une seule et même personne, on peut admettre que la particule est également le support de l'attitude modale de $\mathrm{L}$. Cependant, le fait que $\mathrm{L}=1$ n'implique pas systématiquement une même attitude modale. Ainsi, dans l'expression du regret par exemple, l'attitude de L peut se distinguer de celle de $1 .$.
} 
En ce sens, on pourrait envisager d'interpréter dire mais comme un seul prédicat (que l'on pourrait dans ces exemples gloser en protester ou s'indigner), où mais fonctionnerait comme un adverbe de manière modifiant le verbe, la portée de la particule s'étendant ainsi à l'ensemble du DCé.

En 3, c'est la présence de que entre dire et mais qui rend une telle interprétation plus difficile.

Alors que la pause et la prosodie tendent, comme en 2, à inclure mais au segment introducteur, que contribue à renforcer le décrochement énonciatif. De plus, dans le DR, l'énonciation du verbe introducteur sur une plage basse a pour effet de conférer une certaine autonomie au personnage (Morel et Danon-Boileau, 1998, p. 133). Le locuteur, en se distançant du DCé, lui donne ainsi (plus ou moins consciemment) une apparence d'objectivité.

Le segment introducteur reste neutre jusqu'à la particule. Si mais a encore le rôle descriptif de manifester l'attitude de 1 (réaction négative, indignation), au niveau de la situation représentée, c'est la valeur informative de c- qui déclenche la réaction en d-.

L'attitude modale attribuée à 1 appuie donc l'argumentation du discours global de L, plus qu'elle ne contribue à représenter une attitude dialogique. Cette fonction des particules au sein du DR n'est vraisemblablement pas une spécificité camerounaise. Néanmoins, les rapports qu'elles entretiennent avec les autres indices permettent d'émettre quelques hypothèses quant à une organisation syntaxique du DR qui ne se retrouve pas, nous semble-t-il, dans d'autres corpus de français oral (voir les différences avec les exemples québécois présentés par Vincent, 1989).

\section{Proposition syntaxique}

L'exemple suivant comporte deux occurrences de DR, la seconde étant une reprise de la première. Lors de l'habituelle discussion de fin de journée entre les membres de la famille, CB expose son point de vue dans le débat autour de l'esclavage.

L'observation des procédés de reformulation nous permettra de préciser le rôle des différents indices dans la mise en place du DR.

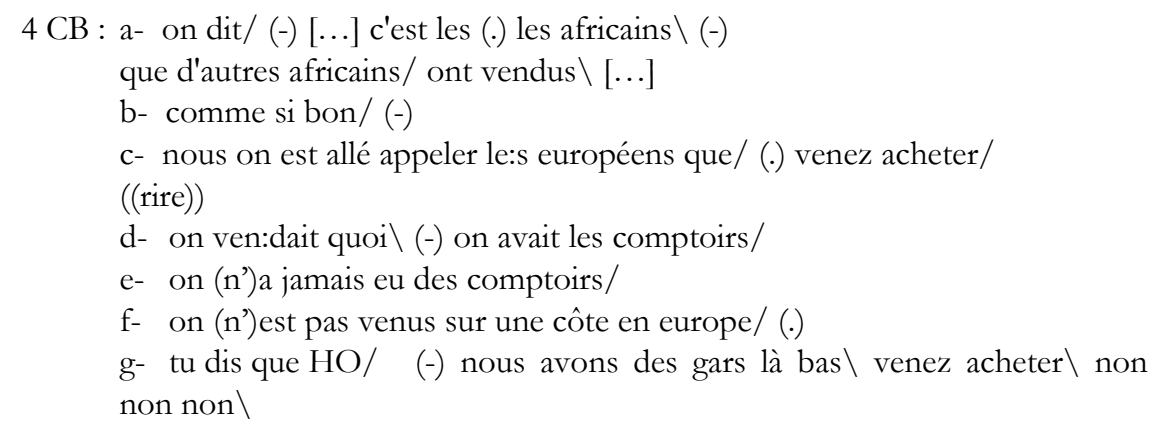

Le DR est explicitement soumis à l'entière subjectivité de L, étant donné son statut fictif indiqué par comme si. Le segment introducteur en c- est élaboré et contient plusieurs informations concernant la situation représentée $: l_{1}$ et $l_{2}$ sont définis (nous, les 
européens), le verbe introducteur est plus lexicalisé que le marqueur neutre dire. Le rire indique la fin du DR et le début de l'argumentation à proprement parler, par la succession de modalités interrogatives et négatives.

Le DR en g- apparaît formellement très différent. La transformation d'une occurrence à l'autre présente des opérations de neutralisation du segment introducteur : $l_{1}$ devient tu (qui ne réfère pas à l'un des interlocuteurs de $\mathrm{L}$, mais fonctionne ici comme un indéfini), $1_{2}$ n'est pas explicite. Le sens du verbe introducteur de c-, appeler, est restitué en g- par le marqueur neutre dire et la particule $\mathrm{HO} /$.

D'une part, le parallélisme évident entre appeler... que et dis que $\mathrm{HO} /$, et la pause nette qui suit la particule inviteraient à considérer celle-ci comme appartenant au segment introducteur. "Quelle que soit l'élaboration formelle de la séquence, le dit reste argument du prédicat de dire »(Ploog, 2004, p. 305). Ici, la pause nous semble indiquer la frontière entre le dire, l'ensemble verbe introducteur/particule, et son argument, le dit. "Son emploi massif, voire quasi-systématique entre discours citant et discours cité ou entre discours citant et le que introducteur du DR est [...] consubstantiel à l'acte de mise en forme du DR» (Queffélec, 2006, p. 270). L'auteur, à la suite d'Italia, considère la pause comme le principal indice de DR (il s'agit de corpus respectivement camerounais et gabonais). D'autre part, le statut expressif de l'interjection et sa position à gauche de que semblent la rapporter au discours cité.

La frontière entre le dire et le dit ne recouvre donc pas systématiquement le passage du discours de L à celui de 1 . Les propriétés syntaxiques de que sont transférées à la pause, au profit du maintien d'une structure de surface du DR proche du standard. La succession verbe + que + particule + pause constitue une zone où s'entremêlent les voix de $\mathrm{L}$ et 1 : la particule intègre le DCé (elle est donnée comme verbalisée par l), mais également le segment introducteur, constituant avec le verbe une seule unité prédicative.

L'occurrence de DR suivante, représentant certes un cas rare dans le corpus, va néanmoins dans ce sens. Elle intervient lors d'une conversation très animée entre trois jeunes gens, amis et collègues, en désaccord sur un événement ayant eu lieu la veille.

5- MU : demain/ quand tu vas encore venir dire/ ah: \que mon portable $\backslash$ on va te chassER//

Les adverbes demain et encore sont des connecteurs qui ancrent le DR au discours global dans un cadre temporel pour le premier, et situationnel (évènement similaire à celui sur lequel porte la conversation) pour le second. L'interjection, si elle peut être interprétée comme marqueur de prise de parole, ne participe pas à une structure dialogique, mais plutôt à l'intégration du DR au discours global, encore + dire ah ayant la capacité anaphorique de synthétiser une partie du co-texte (l'interlocuteur de MU s'était plaint d'un vol avant de retrouver finalement l'objet incriminé).

La solidarité du verbe de parole et de la particule est soulignée au niveau phonologique ([dira:]), prosodique (intégration de dire ah: sous une même courbe, montante sur dire, descendante sur ah, et plate sur l'allongement vocalique), mais également par l'ordre des constituants : que occupe la position où l'on avait trouvé une pause dans les exemples 2,3 et 4 . Il est réinvesti de sa capacité à signaler la rection 
entre le dire et le dit, qui coïncide avec la frontière entre le discours de L (dire ab pouvant signifier se plaindre, crier...) et 1 (mon portable).

L'introducteur neutre dire est donc susceptible d'être modifié par le biais de particules apportant des informations complémentaires dont l'interprétation s'étend à l'ensemble de l'énoncé rapporté. Peut-on aller jusqu'à faire l'hypothèse que la coexistence de séquences telles que dire que mais, dire mais, dira ah que... pourrait témoigner d'un processus de grammaticalisation en cours?

Le jeu de présence/absence de que pourrait difficilement relever des notions de juxtaposition/subordination, dont Ploog a montré à quel point elles étaient inopérantes en contexte non-standard. Si l'on admet le fait que la particule puisse être intégrée au segment introducteur, le DCé étant sous la rection de cet ensemble, les occurrences 3 et 4 indiqueraient que la présence de que ne relève pas d'un marquage syntaxique (à ce titre, la pause semble plus structurante), mais plutôt énonciatif. La possibilité de le placer après le segment verbe/particule constituerait ainsi le stade le plus avancé vers une grammaticalisation. Toutefois, cette hypothèse qui peut paraitre séduisante nécessite un examen plus approfondi, tenant compte de facteurs tels que le type de discours rapporté (auto-citation, narration d'un événement passé, hypothétique, fictif...), la nature de la situation et sa formalité, éventuellement la comparaison avec des corpus plus anciens ou provenant d'autres situations. La confrontation à des corpus de français en Afrique, comme d'autres zones de la francophonie permettrait de mieux déterminer dans quelle mesure il s'agit là d'une particularité camerounaise, et si oui quelle est précisément son incidence.

Concernant que, sa fréquence dans notre corpus pourrait s'expliquer par le marquage récurrent du décrochement énonciatif. Cette interprétation pourrait être étendue, au delà du discours rapporté, à d'autres emplois de que. Il pourrait y avoir là le signe, entre deux énoncés, d'une différence de prise en charge, de responsabilité du locuteur par rapport à son propos. 


\section{RÉFÉRENCES BIBLIOGRAPHIQUES}

Auchlin, A. (1981), « Réflexions sur les marqueurs de structuration de la conversation », Etudes de Linguistique Appliquée, n 44, 88-103.

AuthiER, J. (1978), «Les formes du discours rapporté », DRL $A V, \mathrm{n}^{\circ} 17,1-88$.

Faure, L. et Verine B. (2004), "Authentifier un discours autre en y mettant du sien : les vocalisations ah et oh en frontière de discours rapporté direct à l'oral », in Lopez Muñoz, J. M., Marnette, S. \& Rosier, L., (éds), Le Discours rapporté dans tous ses états, Paris, L'Harmattan, 319-327.

GÜLICH, E. (1999), «Les activités de structuration dans l'interaction verbale », in Barberis, J.-M., (éd), le français parlé, variétés et discours, Coll. Praxiling, Université de Montpellier-3, 21-47.

ITALiA, M. (2005), «Variations en fonction de l'âge et du degré de scolarisation dans le français oral des locuteurs gabonais : la question du discours rapporté ", in Ploog K et Rui B., Appropriations du français en contexte multilingue, Éléments pour une réflexion didactique à propos des situations africaines, Besançon, P.U. Franche-Comté, 191-207.

Meunier. A, M.-A. Morel, L. Danon-Boileau Et N. Tournadre (1991), «Intégration discursive et intégration syntaxique », Langages, $\mathrm{n}^{\circ} 104$, p. 111-128.

Morel, M-A. et L. Danon-Boileau (1998), Grammaire de l'intonation, l'exemple du français, Paris, Ophrys, 128-133.

PloOG, K. (2004). "Stratégies et structures du discours rapporté dans les récits abidjanais », in Lopez Muñoz, J. M., Marnette, S. \& Rosier, L., (éds), Le Discours rapporté dans tous ses états, Paris, L'Harmattan, 297-306.

QUEFFELEC, A. (2006), «Restructurations morphosyntaxiques en français populaire camerounais: l'expression des modalités injonctives et interrogatives dans le discours rapporté », $L e$ français en Afrique, revue du ROFCAN, $\mathrm{n}^{\circ}$ 21, Didier-érudition, 267- 280.

VINCENT, D. (1989), "Les particules d'attaque d'énoncés de conversations rapportées », in Weydt H. (éd.), Sprechen mit Partikeln, Berlin, de Gruyter, 592-600.

VinCENT, D. et Dubois, S. (1997), Le Discours rapporté au quotidien, Québec, Nuit blanche.

\section{CONVENTIONS DE TRANSCRIPTION}

\footnotetext{
$/, \backslash$ : intonation montante, descendante

: : allongement vocalique

(.) : micro-pause

$(-)(-)$

$(--) \quad$ : pause courte, moyenne, longue

( ) : séquence incertaine

MAJ : syllabes accentuées

$=\quad$ : enchaînement rapide
} 
